\title{
Multi-frequency, multi-epoch VLBI observations of the M87 jet
}

\section{Kazuhiro Hada ${ }^{1,2}$, Marcello Giroletti ${ }^{1}$, Gabriele Giovannini ${ }^{1,3}$, Akihiro Doi ${ }^{4}$, Motoki Kino ${ }^{4}$, Hiroshi Nagai ${ }^{2}$, Mareki Honma ${ }^{2,5}$ and Noriyuki Kawaguchi ${ }^{2}$}

${ }^{1}$ INAF Istituto di Radioastronomia, via Gobetti 101, I-40129 Bologna, Italy email: hada@ira.inaf .it

${ }^{2}$ National Astronomical Observatory of Japan, Osawa, Mitaka, Tokyo 181-8588, Japan ${ }^{3}$ Dipartimento di Astronomia, Università di Bologna, via Ranzani 1, I-40127 Bologna, Italy

${ }^{4}$ Institute of Space and Astronautical Science, Japan Aerospace Exploration Agency, 3-1-1 Yoshinodai, Chuo, Sagamihara 252-5210, Japan

${ }^{5}$ Department of Astronomical Science, The Graduate University for Advanced Studies (SOKENDAI), 2-21-1 Osawa, Mitaka, Tokyo 181-8588, Japan

\begin{abstract}
We present some of preliminary results obtained by our dense monitoring project of the M87 jet with VERA 22 and $43 \mathrm{GHz}$ starting from October 2010. The aims of this monitor are to clarify the detailed physical properties of the M87 jet base near the black hole, including the connection to $\gamma$-ray productions, jet kinematics and nuclear opacity. We detected a remarkable increase of the radio flux from the jet base of M87 during an elevated very-high-energy $\gamma$-ray activity occurred in the early 2012, suggesting that the $\gamma$-ray is produced in the immediate vicinity of the central black hole.
\end{abstract}

Keywords. instrumentation: high angular resolution, techniques: interferometric, galaxies: active, galaxies: jets

\section{Introduction}

The radio galaxy M87 accompanies one of the best studied AGN jets through radio to very-high-energy (VHE) $\gamma$-ray. Its proximity $(16.7 \mathrm{Mpc})$ and the large black hole $\left(6 \times 10^{9} M_{\odot}\right)$ provides an unprecedented resolution of 1 milliarcsecond $($ mas $)=0.08 \mathrm{pc}=$ 140 Schwarzschild radii $\left(R_{\mathrm{S}}\right)$, allowing us to probe processes of jet formation and $\gamma$-ray productions by directly resolving the associated regions with high-resolution VLBI (Hada et al. 2011, 2012, 2013). Our on-going monitoring observations of M87 with EVN at $5 \mathrm{GHz}$ have revealed detailed structural evolutions of this jet and their possible connection to VHE $\gamma$-ray activities, especially for the peculiar feature HST-1 which is located at $\gtrsim 120$ pc from the nucleus (Giovannini et al. 2010; Giroletti et al. 2012). On the other hand, the rapid (day-scale) VHE $\gamma$-ray variabilities constrain a horizon-scale volume $\left(\lesssim 10 \delta R_{\mathrm{S}}\right)$ of the emitting site, suggesting that the nucleus of M87 itself is an alternative candidate of the high-energy events (Aharonian et al. 2006; Acciari et al. 2009).

\section{M87 monitor program with VERA at 22 and $43 \mathrm{GHz}$}

To investigate a possible connection to $\gamma$-ray events and also detailed structural/flux evolutions near the black hole, we started a dense ( biweekly) monitoring program of the M87 jet base using VERA 22 and $43 \mathrm{GHz}$ from October 2010. In Figure 1 (left), we show examples of VERA images of M87 at 22 and $43 \mathrm{GHz}$. Generally, the VERA array is less sensitive to the extended jet emission due to the lack of short baselines, but is able 

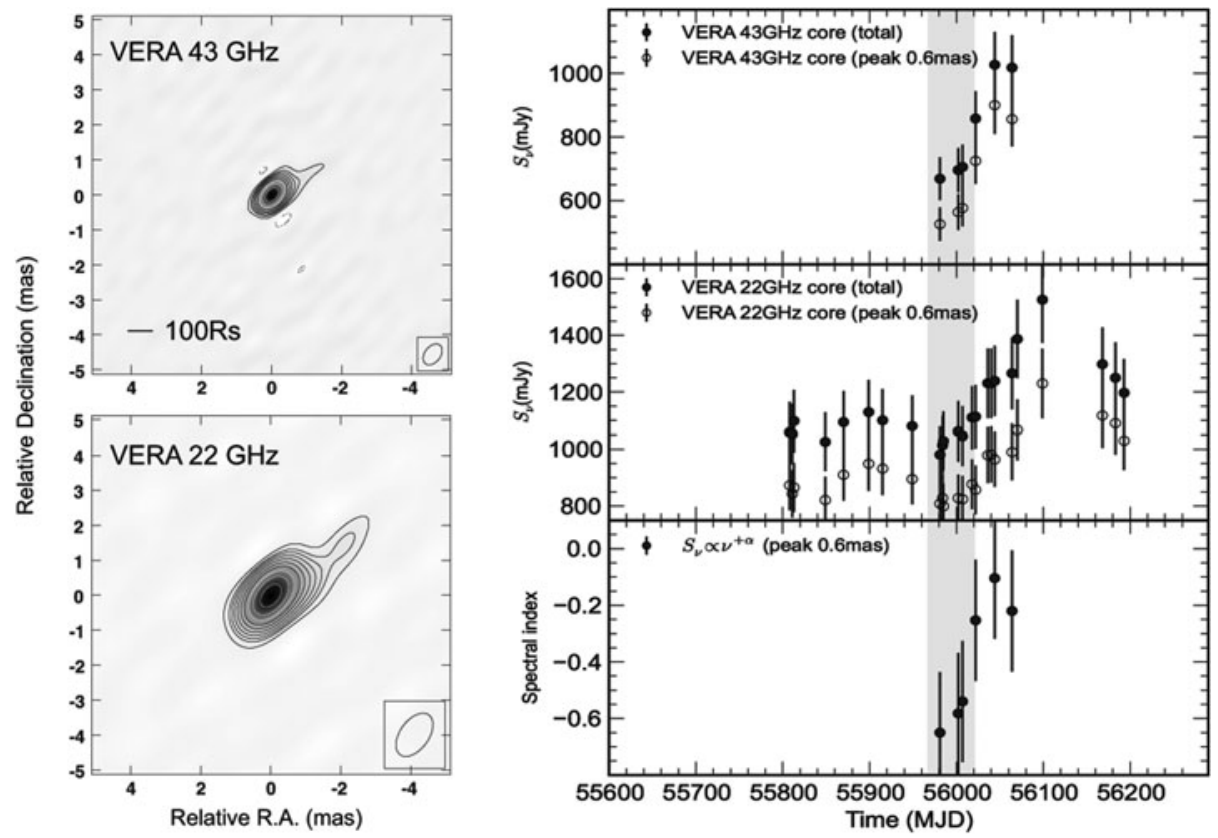

Figure 1. VERA images of the M87 jet base at 43 and $22 \mathrm{GHz}$ (left). Radio light curves of the M87 core at 22 and $43 \mathrm{GHz}$ from September 2011 to September 2012, and an evolution of the spectral index between 22 and $43 \mathrm{GHz}$. A vertical shaded area indicates a period of the elevated VHE activity in 2012 reported by Beilicke et al. (2012).

to resolve the innermost region down to $\sim 60 R_{\mathrm{S}}$ thanks to its high-resolution capability. In Figure 1 (right), we show some of the obtained light curves (at 22 and $43 \mathrm{GHz}$ ) of the M87 core from September 2011 and September 2012. During this period, VERITAS reported an elevated VHE activity from M87 (Beilicke et al. 2012). Coincident with this event, we detected a strong flux increase from the radio core at both 22 and $43 \mathrm{GHz}$. These results suggest that the VHE activity in 2012 originates in the jet base which is in the immediate vicinity of the black hole. Moreover, we discovered a clear frequencydependent evolution of the radio flare and a time-lag between 22 and $43 \mathrm{GHz}$ (as seen by the continuous change of the spectral index), which is evidence a nuclear opacity effect. These monitoring projects with VERA and also EVN are actively going on. We are also conducting high-accuracy core shift measurements with the VERA dual-beam astrometry technique.

\section{Acknowledgments}

The VERA is operated by Mizusawa VLBI Observatory, a branch of National Astronomical Observatory of Japan.

\section{References}

Aharonian, F., Akhperjanian, A. G., Bazer-Bachi, A. R., et al. 2006, Science, 314, 1424

Acciari, V. A., Aliu, E., Arlen, T., et al. 2009, Science, 325, 444

Beilicke, M., and VERITAS Collaboration 2012, arXiv 1210:7830

Giovannini, G., Casadio, C., Giroletti, M., et al. 2010, arXiv 1006.3243

Giroletti, M., et al. 2012, A\&A, 538, L10

Hada, K., Doi, A., Kino, M., et al. 2011, Nature, 477, 185

Hada, K., Kino, M., Nagai, H., et al. 2012, ApJ, 760, 52

Hada, K., Kino, M., Doi, A., et al. 2013, ApJ, 775, 70 